Natures Sciences Sociétés 19, 211-212 (2011)

(C) NSS-Dialogues, EDP Sciences 2011

DOI: $10.1051 / \mathrm{nss} / 2011164$
Disponible en ligne sur :

www.nss-journal.org
Natures Sciences Sociétés

Éditorial

\title{
Heidelberg, vingt ans après
}

En 1992 (juste avant le lancement de la revue NSS), le rapport Brundtland débouchait sur la conférence de Rio et sur un agenda ambitieux autour de l'idée de développement durable, suscitant une prise de conscience généralisée - du citoyen à l'ONU -, mais aussi des actions coordonnées à l'échelle mondiale et des programmes de recherche.

Immédiatement, à l'initiative de Michel Salomon, exdirecteur des relations publiques d'un laboratoire pharmaceutique et fondateur de l'ICSE (International Center for a Scientific Ecology) - centre apparemment en dormance actuellement-, un groupe de chercheurs lançait l'appel d'Heidelberg. Signé par de nombreux spécialistes de diverses disciplines, auréolés de distinctions fameuses, cet appel revendiquait une écologie "scientifique» positive et dénonçait une idéologie irrationnelle en ce qu'elle s'oppose au progrès scientifique et industriel et travestit, implicitement, la discipline de l'écologie. Ces chercheurs exprimaient ainsi la vieille revendication scientiste selon laquelle seule la science sait répondre aux besoins de la société.

Vingt ans après le Sommet de la Terre, « Rio + 20 » se prépare à tous les niveaux : gouvernements, chercheurs et ONG - pour ces dernières avec intensité, dans la foulée des forums altermondialistes.

Dans le laps de temps séparant les deux « Rios », s'est de plus en plus imposée l'idée d'une nécessité pour la recherche d'en passer par l'interdisciplinarité et de créer des formes de collaboration entre chercheurs et acteurs, aussi bien pour poser les bonnes questions de recherche à propos du développement durable que pour les résoudre. NSS s'est inscrite dans ce mouvement, se l'est approprié, a contribué à en affiner les concepts, les méthodes et le savoir-faire et en a publié certains résultats.

C'est pourquoi le lancement de la fondation Écologie d'avenir ne peut nous laisser indifférents. En faisant appel au même réseau de scientifiques - on pourra comparer utilement la liste des premiers signataires en 1992 et la composition du conseil d'orientation de la fondation -, ce nouvel organisme reprend les positions de l'appel d'Heidelberg pour promouvoir l'« écologie positive » (expression qui joue habilement sur la confusion entre la discipline scientifique, les actions dans le domaine de l'environnement et l'écologie politique).

Pour la fondation, « l'écologie positive est réparatrice, elle éduque au lieu de culpabiliser, elle invente et propose au lieu d'interdire "; elle est basée sur le progrès scientifique et technologique. Elle est le lieu pour « repenser les relations entre science, économie et protection de l'environnement » ( $c f$. site internet d'Écologie d'avenir).

Une exégèse de ce court texte fondateur met en relief plusieurs éléments :

- une autonomie sous surveillance pour l'écologie, qui est alors plus un champ d'application des autres disciplines qu'une discipline propre ;

- l'absence d'analyse des interactions entre sciences de la nature et sciences de l'homme et de la société ;

- une conception des rapports entre sciences et sociétés basée sur le vieux modèle de l'ingénierie (dominance de la technologie, absence des acteurs...).

On peut toutefois noter une grande différence avec Heidelberg : les agents économiques, autrefois en sousmain, apparaissent désormais comme partenaires actifs de la fondation (la structure d'une fondation est favorable à la mobilisation de ressources financières très diverses). On est passé d'une défense de la belle Science à la promotion d'une action coordonnée entre grandes entreprises et recherche.

Cette entrée en scène des sociétés du CAC 40 peut, bien sûr, être banalisée et réduite à un greenwashing de plus dans l'attirail de la légitimation écologique, mais, idéologiquement, c'est le subtil mélange entre «la science par-dessus tout » et "la création de nouveaux marchés » qui domine et qui sera à coup sûr utilisé dans les actions de la fondation, comme dans sa communication, en particulier dans le contexte de Rio +20 .

Il est évidemment inacceptable qu'en 2011, l'Académie des sciences ait couvert une telle initiative (voir 
l'article d'Hervé Kempf dans Le Monde du 27 septembre 2011) qui remet au premier plan le débat sur les survivances d'un scientisme éculé, sur ses effets pervers sur le fonctionnement de la science actuelle, et sur une imbrication de plus en plus nette entre recherche publique et grandes entreprises.

NSS aurait pu ignorer ce "non-événement" scientifique (par ailleurs entaché par la personnalité de son fondateur, Claude Allègre, et par d'autres débats), mais ne peut négliger l'arrivée de ce nouveau protago- niste " institutionnel », même si la place qu'il occupera dans la recherche française reste incertaine. Cela doit nous inciter, dans la revue NSS comme à l'association NSS-Dialogues, à la vigilance et à accentuer nos actions sur les deux grands chantiers que sont l'interdisciplinarité et les rapports sciences-sociétés, qui constituent, depuis le début, le cœur de nos orientations stratégiques.

Claude Millier 